\section{Combined In Vitro and In Vivo Propagation for Rapid Multiplication of Grapevine cv. Arka Neelamani}

\author{
Pious Thomas ${ }^{1}$ and John W. Schiefelbein \\ Department of Biology, University of Michigan, Ann Arbor, MI 48109-1048
}

\begin{abstract}
Additional index words. acclimatization, micropropagation, pruning, rejuvenation, stem cuttings, Vitis vinifera
\end{abstract}

\begin{abstract}
A novel combination of in vitro and in vivo approaches was employed to generate sufficient stock of an introduced grape (Vitis vinifera L.) cv. Arka Neelamani which significantly accelerated the multiplication rate. The in vitro part included induction of root and shoot growth in shoot tip and nodal microcuttings in MS medium containing $1 \mu \mathrm{M}$ IAA, sequential pruning of shoots at $1,1.5$, and 2 months, leaving the basal one to two nodes, resulting in fresh sprouts on the stump, and use of remaining stumps for in vivo establishment. The in vivo part included acclimatization of in vitro rooted plantlets and stumps, use of single node cuttings from 1.5- to 2-month-old in vivo shoots for the subsequent propagation, and utilizing the fresh sprout growth from these cuttings and in vivo stumps for further propagation. Employing both in vitro and in vivo approaches, we achieved a multiplication rate unparalleled to the general micropropagation or conventional propagation and significant stock was obtained within 6 months of introducing the material. The in vivo plants exhibited adult characters like distichous phyllotaxy, three lobed leaves and normal pattern of tendril development within 2 months from planting.
\end{abstract}

Grapevine is conventionally propagated by using dormant hardwood cuttings ( 36 to 46 $\mathrm{cm}$ long) collected from well-developed current season's canes during the winter. They are planted in the nursery during the spring, and one season's growth gives plants to transplant to the vineyard (Hartmann et al., 1997). This is a season-bound slow process for the multiplication of new cultivars, elite types or exotic introductions. Micropropagation has offered great potential for rapid propagation of grape (Cao, 1990; Hartmann et al., 1997; Monette, 1988). One criticism of micropropagation in grape is the possibility of carrying the in vitro induced rejuvenation effect to the field, a phenomenon that may persist for years (Deloire et al., 1995; Fournioux and Bessis, 1993; Martinez and Mantilla, 1995). Micropropagation in grape is accomplished either through induction of multiple shoots from nodal explants in a cytokinin-supplemented

\footnotetext{
Received for publication 19 Apr. 2000. Accepted for publication 28 Feb. 2001. P.T. acknowledges the award of Overseas Associateship by the Dept. of Biotechnology, Ministry of Science and Technology, Government of India, which facilitated the exchange visit program. We thank Peter Kaufman and Larry Noodén for reviewing the manuscript. The supply of the grape culture from the Indian Institute of Horticultural Research (ICAR), Bangalore, India, is acknowledged. The cost of publishing this paper was defrayed in part by the payment of page charges. Under postal regulations, this paper therefore must be hereby marked advertisement solely to indicate this fact.

${ }^{1}$ Current address: Division of Biotechnology, Indian Institute of Horticultural Research, Hessaraghatta Lake, Bangalore -560 089, India. E-mail address: piousthomas@usa.net
}

medium followed by the rooting of shootlets (Barlass and Skene, 1980; Heloir et al., 1997; Lee and Wetzstein, 1990) or induction of both root and shoot growth in microcuttings using an auxin supplemented medium (Cao, 1990; Thomas, 1997, 1998a). The latter approach is closer to the natural mode of propagation using cuttings, and has advantages including reduced chance for induction of variation in vitro, use of single medium formulation for both multiplication and rooting, possibility of keeping the stock cultures for years without the loss of multiplication potential with serial subcultures, avoidance of hyperhydricity, and most importantly, return of normal plants with adult characteristics to the field (Cao, 1990; Thomas, 1997, 1999). Induction of rooting in leafy greenwood cuttings with bottom heating and mist for increasing scarce planting material such as virus indexed stocks is also practiced in grapes (Hartmann et al., 1997).

A need for rapid bulking of an exotic cultivar of grape introduced to the United States for research purposes was evident in order to obtain a large amount of tissue from a few introduced in vitro plantlets for use in a gene expression study. The study was undertaken to develop methods for rapid propagation of an introduced cultivar within the shortest possible time employing a combination of in vitro and in vivo approaches.

\section{Materials and Methods}

Plant material and culture conditions. An in vitro culture of grape cultivar Arka Neelamani formed the starting material for the study. This culture was initiated 6 years beforehand and its description can be found elsewhere (Thomas, 1997, 1999, 2000). The introduced initial stock of four plantlets each with three to four nodes was transferred to fresh medium using shoot tip or leaf-bearing single node microcuttings. The medium was comprised of MS (Murashige and Skoog, 1962) constituents, $3 \%$ sucrose, $1 \mu \mathrm{m}$ indole : 3 acetic acid (IAA) and $0.25 \%$ phytagel. Sigma baby food jars with $35 \mathrm{~mL}$ medium and three microcuttings or Magenta boxes (Sigma Chemical Co., St. Louis) with $50 \mathrm{~mL}$ medium and four microcuttings were employed. The cultures were incubated at $26 \pm 2{ }^{\circ} \mathrm{C}$ under $16 \mathrm{~h}$ photoperiod $\left(40-50 \mu \mathrm{mol} \cdot \mathrm{m}^{-2} \cdot \mathrm{s}^{-1}\right)$ provided by coolwhite fluorescent tubes.

In vitro stock bulking. After 3 to 4 weeks, new shoots formed on the above microcuttings were pruned, leaving one to two basal nodes, and the severed sprout served as stock for additional microcuttings. The fresh sprouts (one to two) formed on the stump were severed after $\approx 2$ weeks and the stumps were left for the next round of sprouting. The stumps after one to three cycles of in vitro pruning were used for in vivo planting. This approach was followed for 2 to 4 months from the time of introduction to build up in vitro stock.

In vivo establishment of plants. For in vivo establishment/acclimatization, 3- to 4-weekold directly rooted microcuttings with 2 to 5 $\mathrm{cm}$ shoot growth, or stumps with one to two basal nodes after one to three rounds of in vitro pruning, were used. The roots were washed to remove any adhering medium and then individually planted in Sunshine Mix 1 (Sungrow Horticulture, Washington, D.C.) in $8 \times 8 \times 7.5$ $\mathrm{cm}$ plastic pots arranged in 100-K flats (Kord Products, Ontario, Canada) and covered with a transparent dome. These were incubated under the same conditions as the stock culture except for higher light intensity (60-80 $\mu \mathrm{mol} \cdot \mathrm{m}^{-2} \cdot \mathrm{s}^{-1}$ ) with light source $\approx 50 \mathrm{~cm}$ above or in a Conviron chamber under similar conditions. The domes were partly opened after 1 to 2 weeks and fully removed after 3 weeks. In a subsequent experiment, the stumps were used for ex vitro planting either directly after pruning or a week later when the new sprouts were just emerging. Observations on percentage of establishment and sprout growth were recorded after 1 month.

In vivo stock bulking. One and a half or two months after in vivo planting when the shoots grew $\approx 20-30 \mathrm{~cm}$ tall, shoot tip $(2-3 \mathrm{~cm})$ and single node leafy cuttings $(2-5 \mathrm{~cm}$ stem) were prepared from them. In a preliminary trial, the basal ends of these cuttings were given a 10 -min pre-planting dip in IAA $(100 \mu \mathrm{M})$, prepared in MS medium with 3\% w/v sucrose. Based on the results from this trial, the cuttings in the next experiment were segregated into three categories: shoot tip cuttings, upper nodal tender cuttings, and lower nodal softwood cuttings. Four cuttings were planted per pot $(8$ $\times 8 \times 7.5 \mathrm{~cm})$ in each category, which constituted one replication and there were eight replications per treatment. The incubation conditions for the cuttings were similar to those provided during acclimatization of in vitro derived plantlets or stumps. Observations on survival of the cuttings, percentage of healthy plant output and shoot height were recorded 
at 4 weeks after planting and this experiment was repeated twice. This was followed by an experiment in which the lower nodal softwood cuttings were given a pre-planting dip treatment with IAA or indole 3 butyric acid (IBA, $100 \mu \mathrm{M})$. There were eight replications per treatment with four cuttings per replication and this experiment was repeated once.

Experiments were designed in a completely randomized format with six to eight replications per treatment to provide sufficient error degrees of freedom. The data were subjected to single factor analysis of variance employing MS Excel 98 Macintosh edition. Mean SE values are presented.

\section{Results and Discussion}

A depiction of combined in vitro and in vivo approaches for multiplication of grapes can be found in Fig. 1. The in vitro multiplication included in vitro pruning, use of new sprouts for subculture, and use of the stumps after sequential pruning for in vivo establishment whereas in vivo multiplication included the use of single node leafy cuttings.

In vitro stock multiplication. The shoot tip and nodal microcuttings generated single shoots subsequent to rooting and two to five microcuttings $($ mean $=3.2)$ could be harvested from these shoots after 1 month (Fig. 2A). The shoot growth and yield of microcuttings depended on the weight, leaf size or position on the stock shoot of the cutting (Thomas, 2000). Upon pruning or removal of the sprout, one to three new sprouts developed from the stump (mean $=1.2 \pm 0.1)$, giving one to five additional cuttings (mean $=2.5 \pm 0.2$ ) per pruned stock within 2 weeks after pruning (Fig. 2B). This saved the time and energy resources for the rooting process in a freshly planted microcutting. The shoot growth on pruning depended on the vigor of the stock culture. Cuttings with vigorous roots, particularly those with relatively larger lamina, were more responsive than others with less vigorous roots. The second pruning yielded one to three (mean $=2.0 \pm 0.2$ ) cuttings, whereas the next pruning was not rewarding. Coupled with two repeated prunings, an original microcutting gave 8 to 12 new microcuttings within 2 months, besides yielding another three to five microcuttings from each of those cuttings collected and cultured at the 1 month stage. Thus at the end of 2 months, in vitro pruning yielded $\approx 2.5$-fold more microcuttings compared to direct subculturing at monthly intervals. The high propagation rate thus attained in the early stage was proportionately transmitted to the next subculturing cycles resulting in an enormous multiplication potential compared to normal micropropagation. Employing this approach, we generated a good in vitro stock within 3 to 4 months of introducing this cultivar. In vitro pruning is simple in operation and is resource saving-medium, vessel, time, and manpower-compared to subculturing at regular intervals.

In vivo establishment of plants. Using 3- to 4-week-old rooted plantlets or one to two noded stumps after one to three in vitro

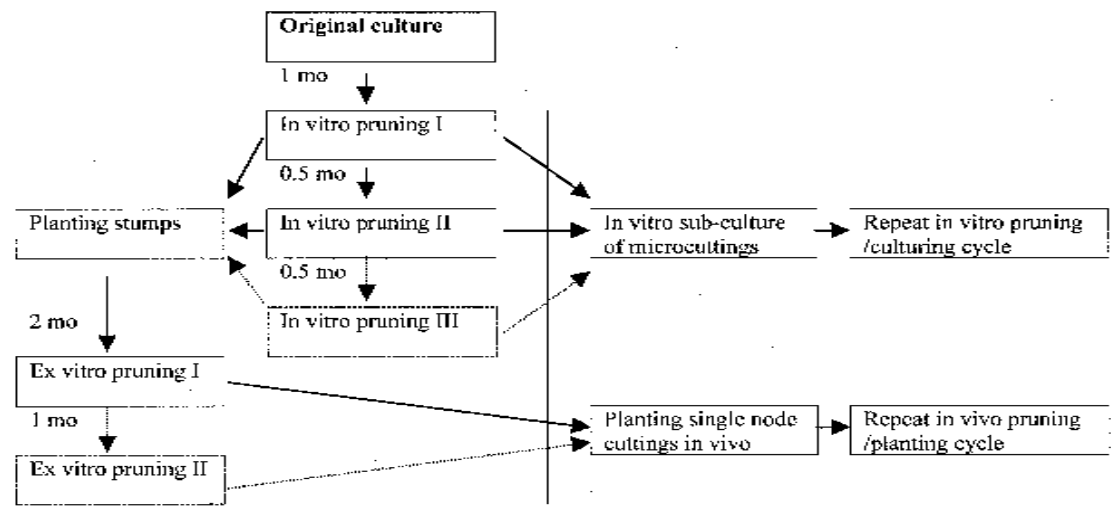

Fig. 1. Scheme for rapid stock bulking of grapes employing combined in vitro and in vivo approaches The scheme takes into account first in vitro pruning at 1 month (mo) yielding three microcuttings on average, two additional prunings at 1.5 and 2 months from the first culture yielding three and two microcuttings respectively, and one stump for in vivo planting at 1.5 or 2 months. In vivo part accounts production of one shoot giving four to six nodal cuttings from the middle and lower parts of the shoot and two to three tender cuttings from the upper region 2 months after planting the stump, additional four to six cuttings from the new shoot after 1 month of first in vivo pruning and the use of single node cuttings for further in vivo multiplication. Activities right to the vertical line are repeat of that on the left. Dotted lines indicate optional activities.
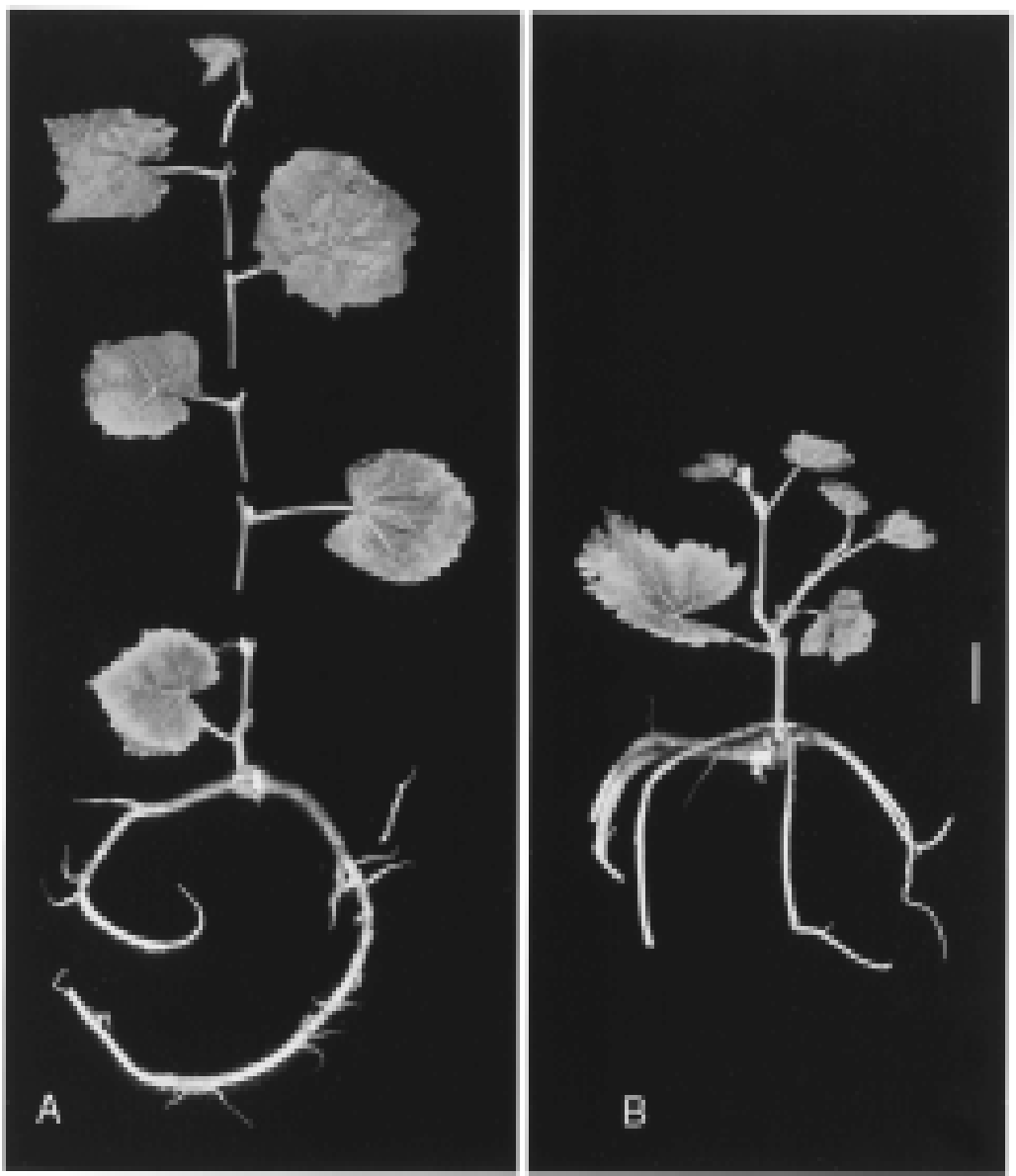

Fig. 2. Plate showing a 1-month-old in vitro plantlet displaying shoot tip and single node microcuttings (A) and a stump with new sprouts 2 weeks after the first pruning $(\mathbf{B}) ; \mathrm{Bar}=1 \mathrm{~cm}$. 
prunings, we obtained $90 \%$ to $100 \%$ establishment for different batches. The stumps showed a reduction in establishment with age (Thomas, 1998b) or more pruning cycles. In order to facilitate the acclimatization process, the domes were slightly lifted after 1-2 weeks and fully removed by 3 weeks. Some amount of desiccation damage to the leaves formed in vitro was encountered, but the leaves formed ex vitro were normal, similar to observations in sachet technique of acclimatization (Thomas, 1998b). Stumps appeared better than whole plantlets in withstanding the transition to ex vitro.

Considering the planting of nonsprouted stumps directly or using presprouted stumps after 1 week of the previous pruning revealed no significant advantage of the latter: $88 \%$ of the in vitro maintained stumps showed sprouting, and a further $8 \%$ showed bud swelling at 1 week after the pruning. By comparison, only $25 \%$ of the directly planted stumps showed sprouting within this 1 week in vivo. However, the advantage of early sprouting in vitro was not reflected in the final performance, as plants derived from both sets appeared quite comparable at 1 month after the onset of the experiment (Table 1). Considering the saving of in vitro space and the vulnerability of the tender sprouts to desiccation damage in the in vivo environment, direct planting was advantageous over the use of presprouted stumps. The use of protrays for acclimatization gave $80 \%$ establishment and this accommodated four times the number of plants in the growth chamber compared to plastic pots. These plants were relatively less vigorous and they could be transplanted to the pots or a nursery after 1 month.

The in vivo established plantlets or stumps gave a shoot $20-30 \mathrm{~cm}$ tall within 1.5 to 2 months of planting. These shoots showed a few closely spaced basal nodes, upper four to eight large leaf bearing nodes with longer internodes, and then a terminal region with one to three tender leafy nodes and the shoot tip. The shoots showed light reddish-green color and adult plant characteristics like distichous phyllotaxy, tri-lobed leaves with nearly closed sinus and normal pattern of tendril production, i.e., a bifid tendril opposite to leaf at two of three nodes in a repetitive manner, usually designated as N0-N1-N2 arrangement (Mullins et al., 1979;Weaver, 1976) by 1.5-2 months after planting (Fig. 3A). In subsequent observations, increasing light intensity by keeping the plants closer to the light source (30 vs. $50 \mathrm{~cm}$ ) or providing continuous light for $24 \mathrm{~h}$ resulted in shoots with closer nodes and reddish pigmentation of stem. The latter was often associated with in vitro propagated plants in field (Martinez and Mantilla, 1995). Plants in which the roots were pruned at planting (Thomas and Ravindra, 1997) often showed pigmentation of stem and petiole, whereas those planted with intact in vitro roots exhibited largely green parts.

Performance of in vivo-derived cuttings. The shoot tips and the tender upper nodal cuttings exhibited poor survival $(22 \%$ and $50 \%$, respectively) compared to lower nodal softwood cuttings $(91 \%$; difference significant at $P<0.01)$ at 1 month after planting. The output of plantlets from these three categories at this stage was $19 \%, 44 \%$, and $81 \%$, respectively $(P<0.01)$ and the shoot or sprout heights were $5.9,3.3$ and $9.9 \mathrm{~cm}$, respectively $(P<0.05)$. The results from three successive experiments suggested that the softwood cuttings derived from the middle periphery of the stock shoots were best for in vivo multiplication, while shoot tip and tender upper nodal cuttings could be used if one is attempting to exploit maximum number of cuttings. The influence of the position of cutting on the stock shoot was evident from these results. Such positional effects have been documented in other plants (Dick et al., 1998; Wilson, 1993).

It was essential to avoid desiccation damage to the leaf of the cuttings. Otherwise, cuttings either failed to establish or showed poor shoot vigor, depending on the extent of leaf damage. The leaf was vulnerable to desiccation during the first 2-3 weeks, and therefore, incubation under a humid environment was necessary during this period.

In the trial when IAA and IBA were compared, using softwood cuttings, the choice of growth regulator did not significantly affect output of plants or shoot growth. The IAA and IBA $(100 \mu \mathrm{M})$-treated cuttings, respectively, showed $88 \%$ and $94 \%$ plantlet output and 12.1 - and $10.3-\mathrm{cm}$ sprout height $(P>0.05$ in both cases). A subsequent study showed comparable percentage of rooting and sprouting in auxin treated and auxin nontreated leafy cuttings suggesting that the growth regulator treatment can be done away with but at the cost of root and shoot vigor (unpublished data). By 2-2.5 months after planting, the plants propagated using leafy cuttings in vivo showed relatively faster growth than plants established from in vivo source. They continued to display

Table 1. In vivo establishment and growth after 1 month of presprouted or directly planted in vitro derived stumps of grape.

\begin{tabular}{lccc}
\hline & \multicolumn{2}{c}{ Type of stumps } & \\
\cline { 2 - 3 } Parameter & Presprouted & Nonsprouted & Significance \\
\hline Establishment (\%) & $92 \pm 8$ & $92 \pm 5$ & NS \\
Sprouts per stump (mean) & $1.2 \pm 0.1$ & $1.3 \pm 0.1$ & NS \\
Sprout height (cm) & $5.3 \pm 0.6$ & $5.7 \pm 0.9$ & NS \\
\hline
\end{tabular}

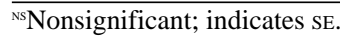
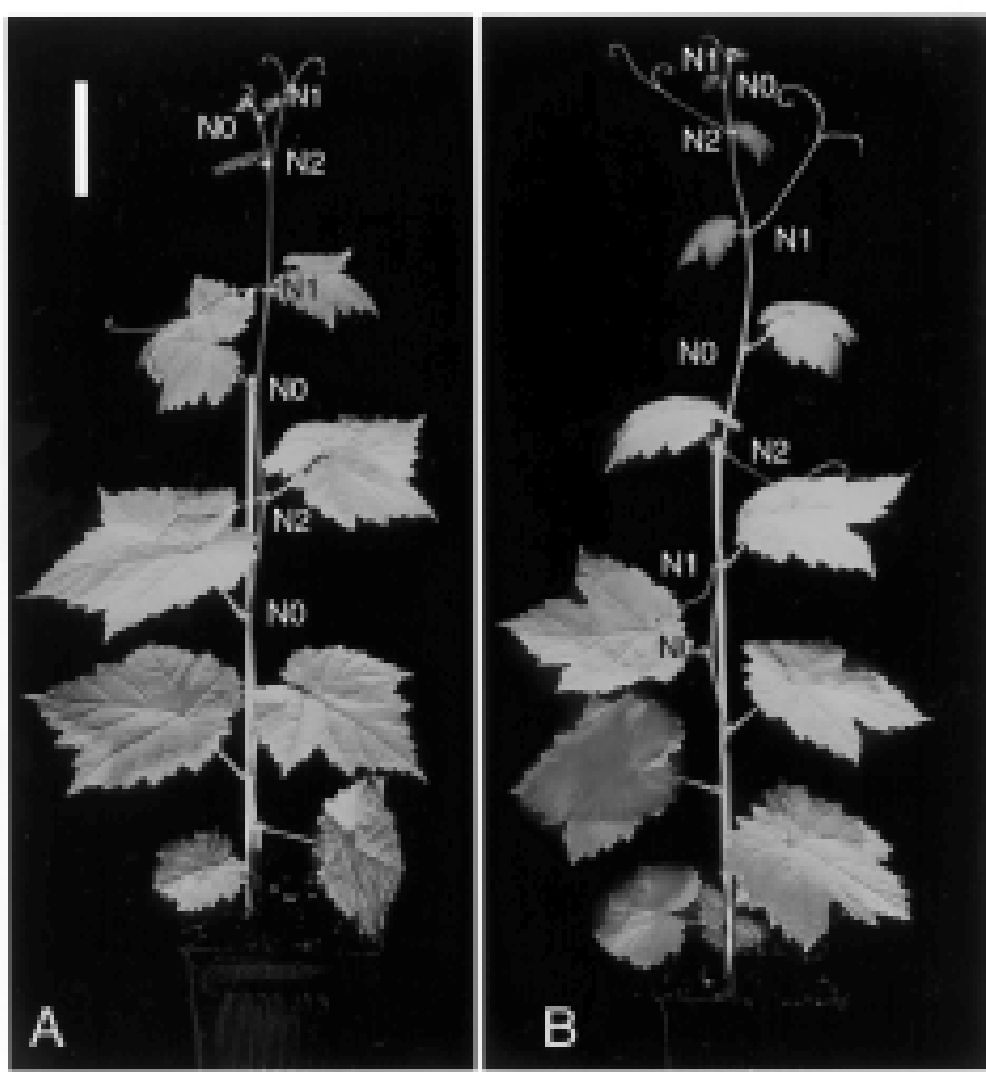

Fig. 3. Two-month-old in vivo plant established either from in vitro stump (A) or from single node leafy cutting from in vivo shoot (B) displaying distichous phyllotaxy, tri-lobed leaves and leaf opposed bifid tendril in N0-N1-N2 pattern $(\mathrm{N} 0=$ tendril lacking node; $\mathrm{N} 1, \mathrm{~N} 2=$ tendril bearing node $) ; \mathrm{Bar}=5 \mathrm{~cm}$. 
adult characteristics including distichous phyllotaxy, tri-lobed leaves and N0-N1-N2 pattern of tendril production (Fig. 3B).

The in vivo stumps that retained the basal region with closer nodes showed sprouting in $\approx 50 \%$ to $60 \%$ cases. In $\approx 50 \%$ of such cases, the new shoot appeared promising giving four to five new cuttings within another 4 to 6 weeks. When the stumps were replanted to fresh growth medium, most of them showed good shoot growth but this practice was labor intensive.

The results presented here demonstrate the feasibility of rapid bulking of elite or rare grape genotypes from limited starting material by employing a combination of in vitro and in vivo approaches. Besides the normal micropropagation approach, which itself is a powerful tool for the rapid multiplication of grapes, pruning of in vitro-formed shoots led to an early high multiplication which in subsequent cycles accelerated the propagation rate many fold (Fig. 1). The in vitro growth was unaffected after several cycles of subculturing unlike reports of multiple shoot induction (Thomas, 1997). This approach makes effective use of stumps which otherwise are discarded. A quick further multiplication was achieved through the use of nodal cuttings from in vivo formed shoots.

The plants in the present study displayed adult plant characteristics including distichous phyllotaxy, tri-lobed leaves, and normal tendril development within 2 months of in vivo planting in contrast to juvenile characters like spiral phyllotaxy, four to five lobed leaves and absence of tendrils (Fournioux and Bessis, 1993; Martinez and Mantilla, 1995; Mullins et al., 1979) in spite of continuous maintenance of the stock in vitro for nearly 7 years. This suggests that the rejuvenation effects in vitro (Deloir et al., 1995; Martinez and Mantilla, 1995; Mullins et al., 1979) are less likely to be transmitted to the field when one resorts to induction of both root and shoot growth from microcuttings using an auxin supplemented medium (Thomas, 1999). The in vitro plants too showed small bifid tendrils which became less frequent and erratic with the period from culture initiation (Thomas, 1999). A recent count of 66 in vitro cultures 2 months into subculture showed small tendril production capability in $\approx 38 \%$ of plants, although deviating from the normal adult pattern. Tendrils and inflorescences are homologous organs and tendrils are considered as slightly differentiated inflorescences (Srinivasan and Mullins, 1978). The in vivo plants in this study appeared uniform with no apparent signs of somaclonal variation except for minor differences in vigor owing to the size of the stumps or cuttings at planting. A field evaluation comparing plants derived from microcuttings vs. multiple shoot induction could verify whether propagation approach influences the transmittance of in vitro induced effects. The combined approach described here is suitable for situations where the initial starting material is sparse as with transgenic clones, newly bred cultivars and introduced germplasm. It can be practiced year-round independent of seasonal effects, with moderate infrastructure facilities. The in vivo incubation conditions described here can easily be simulated in a glasshouse with reduced lighting and occasional misting. Low light intensity during acclimatization and in vivo propagation appeared to be important to avoid pigmentation of stem and leaves and to allow better internode growth facilitating handling of single node cuttings.

\section{Literature Cited}

Barlass, M. and K.G.M. Skene. 1980. Studies on fragmented shoot apex of grapevine. II. Factors affecting growth and differentiation in vitro. $\mathrm{J}$. Expt. Bot. 31:489-495.

Cao, Z. 1990. Grape: Micropropagation. p. 312 328. In: Z. Chen, D.A. Evans, W.R. Sharp, R.V. Ammirato, and M.R. Sondhal (eds.). Handbook of plant cell culture Vol 6, Perennial crops. Academic, New York.

Deloire, A., M. Charpentier, G. Berlioz, A. Colin and G. Gimonnet. 1995. Micropropagation of grapevine: Results of 10 years of experiments in the Champagne vineyard and results of the first vinifications. Amer. J. Enol. Viticult. 46:571578.

Dick, J., F. Magingo, R.I. Smith, and C. McBeth. 1998. Rooting ability of Leucaena leucocephala stem cuttings. Agrofor. Syst. 42:149-157.

Fournioux J.C. and R. Bessis. 1993. Use of carbondioxide enrichment to obtain adult morphology of grapevine in vitro. Plant Cell Tiss. Org. Cult. 31:239-244.

Hartmann, H.T., D.E. Kester, F.T. Davies, and R.L. Geneve. 1997. Plant propagation- principles and practices. Sixth edition. Prentice Hall, N.J.

Heloir, M-C., J-C. Fournioux, L. Oziol, and R. Bessis. 1997. An improved procedure for the propagation in vitro of grapevine (Vitis vinifera cv. Pinot Noir) using axillary bud microcuttings. Plant Cell Tiss. Org. Cult. 49:223-225.

Lee, N. and Y. Wetzstein. 1990. In vitro propagation of muscadine grape by axillary shoot proliferation. J. Amer. Soc. Hort. Sci. 115:324329.

Martinez, M.C. and J.L.G. Mantilla. 1995. Morphological and yield comparison between Vitis vinifera L. cv. Albariño grown from cuttings and from in vitro propagation. Amer. J. Enol. Viticult. 46:195-203.

Monette, P.L. 1988. Grapevine (Vitis vinifera L.), p. 3-37. In: Y.P.S. Bajaj (ed.). Biotechnology in agriculture and forestry, Vol. 6, Crops II. Springer-Verlag, Berlin.

Mullins, M.G., Y. Nair, and P. Sampet. 1979. Rejuvenation in vitro: Induction of juvenile characters in an adult clone of Vitis vinifera L. Ann. Bot. 44:623-627.

Murashige, T. and F. Skoog. 1962. A revised medium for rapid growth and bioassays with tobacco tissue cultures. Physiol. Plant. 15:473497.

Srinivasan, C, and M.G. Mullins. 1978. Control of flowering in the grapevine (Vitis vinifera L.): Formation of inflorescence in vitro by isolated tendrils. Plant Physiol. 61, 127-130.

Thomas P. 1997. Increase in clonal propagation of 'Arka Neelamani' grape (Vitis vinifera L.) through induction of axillaries in in vitro layering technique. Indian J. Agr. Sci. 67:594-596.

Thomas, P. 1998a. Contribution of leaf lamina of grape nodal microcuttings to rooting, root vigour and plantlet growth in vitro. J. Plant Physiol. 153:727-732.

Thomas, P. 1998b. Humid incubation period and plantlet age influence acclimatization and establishment of micropropagated grapes. In Vitro Cell. Dev. Biol. Plant 34:2-56.

Thomas P. 1999. Relationship between tissue growth, $\mathrm{CO}_{2}$ level and tendril formation during in vitro culture of grape (Vitis vinifera $\mathrm{L}$.). Vitis 38:25-29.

Thomas, P. 2000. Microcutting leaf area, weight, and position on the stock shoot influence root vigour, shoot growth and incidence of shoot tip necrosis in grape plantletsin vitro. Plant Cell Tiss. Org. Cult. 61:189-198.

Thomas P. and M.B. Ravindra 1997. Effect of pruning or removal of in vitro formed roots on ex vitro root regeneration and growth in micropropagated grapes. Plant Cell Tiss. Org. Cult. 51:177-180.

Weaver, R.J. 1976. Grape growing. Wiley, New York.

Wilson, P.J. 1993. Propagation characteristics of Eucalyptus globulus ssp. globulus stem cuttings in relation to their original position in the parent shoot. J. Hort. Sci. 68:715-724. 